\title{
EFECTO DEL CONSUMO DE UNA BEBIDA A BASE DE Physalis peruviana, Passiflora edulis, Ananas comosus, Avena sativa, Linum usitatissimum y Stevia rebaudiana, SOBRE EL PERFIL LIPÍDICO Y GLICEMIA, DE MUJERES CON SOBREPESO Y OBESIDAD
}

\author{
Claudia M. Dolores ${ }^{1 *}$, Elena R. Benavides ${ }^{1}$, Óscar O. Osso ${ }^{2}$
}

\begin{abstract}
RESUMEN
Se elaboró una bebida a base de pulpa de Physalis peruviana (12\%), Passiflora edulis (9 \%) y Ananas comosus (9\%), con fibra de Avena sativa (12\%) y Linum usitatissimum (33\%), endulzada con Stevia rebaudiana (1 \%), la misma que cumplió con los parámetros fisicoquímicos, bromatológicos, microbiológicos y sensoriales para ser apta para consumo humano. El estudio del efecto de la bebida fue cuasi experimental en 10 mujeres voluntarias entre los 21 y 39 años de edad, estudiantes de la Facultad de Farmacia y Bioquímica de la UNMSM, todas ellas presentaban sobrepeso u obesidad de acuerdo a su índice de masa corporal y clasificación dictada por la OMS, quienes consumieron la bebida con una frecuencia de cuatro veces por semana durante el periodo de tres meses, con seguimiento mensual de mediciones antropométricas y análisis bioquímicos de triglicéridos, colesterol total, HDL, LDL, glucosa y hemoglobina. Los resultados fueron analizados aplicando t-student para muestras pareadas, con nivel de significancia del valor $\mathrm{p} \leq 0,05$. Obteniéndose resultados estadísticamente significativos respecto a triglicéridos $(\mathrm{p}=0,034)$ y glucosa $(\mathrm{p}=$ 0,002). Por lo que se concluye que la bebida elaborada posee propiedad hipolipemiante en cuanto a triglicéridos y un efecto hipoglicemiante.
\end{abstract}

Palabras clave: sobrepeso, obesidad, fibra, hipolipemiante, hipoglicemiante.

\footnotetext{
* Jr. Puno 1002. Lima 1. Clau.melidolores@gmail.com

${ }^{1}$ Facultad de Farmacia y Bioquímica, UNMSM.

${ }^{2}$ Universidad Nacional José Faustino Sánchez Carrión
} 


\title{
EFECT FROM CONSUMPTION OF A DRINK WITH Pysalis peruviana, Passiflora edulis, Ananas comosus, Avena sativa, Linum usitatissimum and Stevia rebaudiana, ABOUT THE LIPID PROFIL AND GLICEMIA, FROM WOMEN WITH OVERWEIGHT AND OBESITY
}

\begin{abstract}
A pulp-based drink was made from Physalis peruviana (12\%), Passiflora edulis (9\%) and Ananas comosus (9\%), with fiber of Avena sativa (12\%) and Linum usitatissimum (33\%), sweetened with Stevia rebaudiana (1\%), fulfilled the physicochemical, bromatological, microbiological and sensorial parameters to be suitable for human consumption. The study of the effect of the drink was quasi-experimental in 10 volunteer women between 21 and 39 years of age, students of the Faculty of Pharmacy and Biochemistry of the UNMSM, all of them were overweight or obese according to their body mass index and classification dictated by the WHO, who consumed the drink with a frequency of four times a week during the three-month period, with monthly monitoring of anthropometric measurements and biochemical analysis of triglycerides, total cholesterol, HDL, LDL, glucose and hemoglobin. The results were analyzed by applying t-student for paired samples, with level of significance of the $p$ value $\leq 0.05$. Statistically significant results were obtained in terms of triglycerides $(\mathrm{p}=0.034)$ and glucose $(\mathrm{p}=0.002)$. Therefore, it is concluded that the elaborated drink possesses lipid-lowering property in terms of triglycerides and a hypoglycemic effect. Conclusion: Therefore, the elaborated beverage possesses lipid-lowering properties in terms of triglycerides and a hypoglycemic effect was found, but these effects are insufficient for the control of overweight and obesity.
\end{abstract}

Key words: overweight, obesity, fiber, hypolipidemic, hypoglycemic.

\section{INTRODUCCIÓN}

En el Perú, el 62,3 \% de la población, entre los 30 y 59 años, tres de cada cinco, sufre de sobrepeso u obesidad, según estudios del sector $\mathrm{Salud}^{1}$, siendo esta prevalencia mayor en mujeres. La obesidad favorece la expresión de los mismos fenotipos principales descritos en otras formas de resistencia a la insulina, principalmente los de la hipertensión arterial sistémica, la hiperglicemia de ayuno y postprandial, y la dislipidemia caracterizada por elevación de triglicéridos (TG), producción de lipoproteínas de baja densidad (LDL) y reducción de las lipoproteínas de alta densidad (HDL), la obesidad, la dislipidemia y el riesgo cardiovascular que conlleva deberían revertir con dietas hipocalóricas; sin embargo, hasta el momento no hay estudios que lo corroboren ${ }^{2}$; por ello, la elaboración de una bebida, de fácil disponibilidad, que ayude a controlar la dislipidemia, puede contribuir a reducir los índices de riesgo en la salud, por lo que, pensando en las poblaciones con sobrepeso y obesidad, se desarrolló un producto saludable, agradable, inocuo y de fácil disponibilidad, 
con presentación ideal. La bebida elaborada es una alternativa para la industria alimentaria por sus propiedades funcionales, al ser una bebida baja en calorías, recomendable para una alimentación saludable complementando la dieta diaria, con aporte de fibra soluble que disminuya la absorción de lípidos y carbohidratos, que a su vez proteja las paredes del estómago y al hidratarse genere sensación de saciedad al consumidor ${ }^{3}$.

El objetivo de este estudio fue elaborar una bebida con efecto hipolipemiante e hipoglicemiante formulada a base de pulpa de Physalis peruviana (aguaymanto), Passiflora edulis (maracuyá) y Ananas comosus (piña), fibra de Avena sativa (avena) y Linum usitatissimum (linaza), endulzada con Stevia rebaudiana (stevia).

\section{PARTE EXPERIMENTAL}

Materia prima: se utilizó pulpa de aguaymanto, maracuyá y piña, fibra de avena y linaza extraída por proceso térmico en agua a $90{ }^{\circ} \mathrm{C}$ durante 15 minutos, adaptado del método empleado por Thakur ${ }^{4}$.

Insumos: se utilizaron insumos permitidos de acuerdo a las normas nacionales de INDECOPI5 y Codex Alimentarius ${ }^{6}$, tales como: stevia en polvo, agua blanda, carboximetilcelulosa (CMC) y sorbato de potasio.

Equipos y reactivos: propios de laboratorio de alimentos y para análisis bioquímicos.

Tipo de investigación: diseño cuasi experimental de casos y controles.

Población y muestra de estudio: Diez (10) estudiantes mujeres, entre 21 y 39 años de edad con sobrepeso u obesidad, de la Facultad de Farmacia y Bioquímica, de la Universidad Nacional Mayor de San Marcos.

Metodología: Se estableció la formulación apropiada, que cumpla con las especificaciones de la NTP5, el Codex Alimentarius ${ }^{6}$, el análisis proximal de la $\mathrm{AOAC}^{7}$; así como la buena aceptabilidad ante la evaluación sensorial. Siendo esta de base de pulpa de Physalis peruviana (12\%), Passiflora edulis (9\%) y Ananas comosus (9\%), con fibra de Avena sativa (12\%) y Linum usitatissimum (33 \%), endulzada con Stevia rebaudiana (1 \%).

Se les dio a beber al grupo muestral con una frecuencia de cuatro veces por semana y un volumen de $300 \mathrm{~mL}$ por vez. Esta frecuencia se planteó al revisar otras investigaciones en las que se ha observado un efecto hipolipemiante y/o hipoglicemante en pacientes con sobrepeso u obesidad, siendo el tiempo referencial igual o mayor a un mes. Previo a la primera ingesta $\mathrm{y}$ al término del primero, segundo y tercer mes de consumo, se determinaron las medidas antropométricas (peso, talla y perímetro de cintura); así como, los niveles de hemoglobina, glucosa y perfil lipídico (triglicéridos (TG), colesterol total, LDL y HDL). Los resultados fueron analizados aplicando t-student para muestras pareadas, con nivel de significancia del valor $\mathrm{p} \leq 0,05$. 


\section{RESULTADOS Y DISCUSIÓN}

Determinaciones antropométricas: Mediante el test de normalidad Shapiro-Wilk (SW) comparación de muestras relacionadas, en Peso, no cumplió con los criterios de normalidad ( $\mathrm{S}-\mathrm{W}_{\text {Inicio-final }}=0,794 ; \mathrm{p}=0,012$ ), al aplicar test de Wilcoxon no hubo variaciones estadísticamente significativas. En la figura 1 podemos observar la evolución del grupo de estudio en cuanto al peso, en la figura 2 la evolución en cuanto al IMC y en la figura 3 la correspondiente al perímetro de cintura.

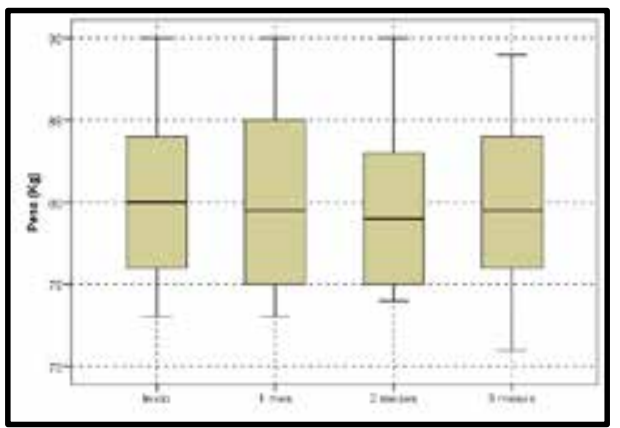

Figura 1. Evolución del grupo de estudio en cuanto al peso.

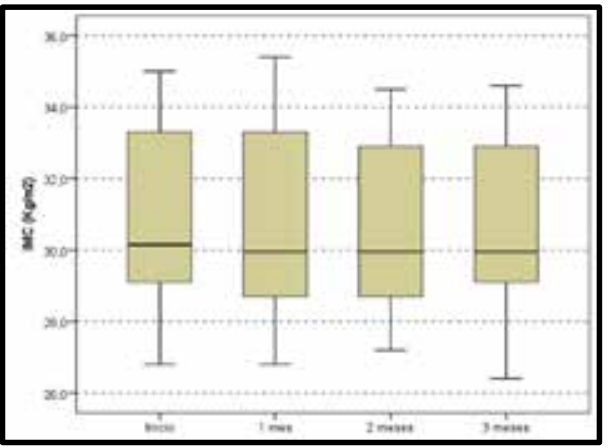

Figura 2. Evolución del grupo de estudio en cuanto al IMC.

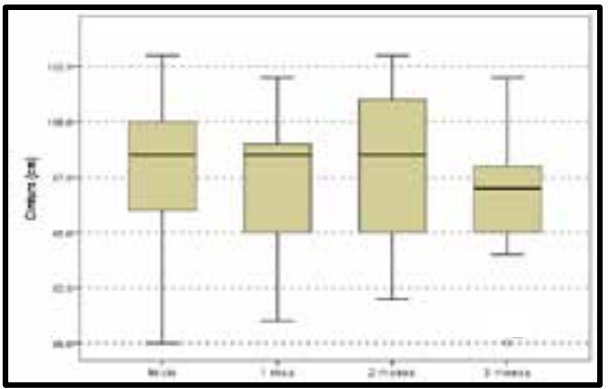

Figura 3. Evolución del grupo de estudio en cuanto al perímetro de cintura. 
Los resultados antropométricos no presentaron diferencia significativa en la disminución de los índices relacionados con el sobrepeso y la obesidad, por lo cual la bebida no posee per se un efecto que ayude a contrarrestar y/o controlar los niveles de obesidad, podemos decir que la razón de los resultados observados es debido a que el grupo de estudio consumió la bebida formulada, pero sin intervención en la dieta, ni en la actividad física; siendo la combinación de estos más efectivo en el tratamiento de la obesidad, que cada uno de forma separada, según la $\mathrm{OMS}^{8}$. Estos resultados son comparables con lo reportado por Alonso ${ }^{9}$, no encontrando diferencias estadísticamente significativas en la disminución del IMC por consumo de licuado de avena con manzana en pacientes con sobrepeso y obesidad, pero en contraste con el presente estudio, sí reportaron una disminución significativa en el perímetro abdominal.

Probablemente el consumo de la bebida podría servir como apoyo en un régimen de pérdida de peso con una dieta saludable y actividad física, lo cual puede evaluarse en investigaciones futuras.

La comparación de los resultados de triglicéridos (TG) aplicando t-student para muestras pareadas, no cumplió con los criterios de normalidad ( $\mathrm{S}-\mathrm{W}$ Inicio-1 mes $=0,931, \mathrm{p}=0,460$; $\mathrm{S}-\mathrm{W} 2$ mes-3 mes $=0,944, \mathrm{p}=0,601$; S-W Inicio-Final $=0,925, \mathrm{p}=0,403)$, excepto entre el primer y segundo mes (S-W 1 mes-2 mes $=0,737, p=0,003)$. Mientras que entre el segundo $\mathrm{y}$ tercer mes existe diferencia estadísticamente significativa, $\mathrm{t}=2,485, \mathrm{p}=0,035$, donde el tamaño del efecto fue de magnitud media $(d=0,786)$ usando los criterios de Cohen ${ }^{10}$; además, la comparación inicial con final del estudio indican una diferencia estadísticamente significativa, $t=2,504, p=0,034$, con efecto de magnitud media $(d=0,792)$.

El consumo de la bebida, entonces, provocó la disminución de los niveles séricos de TG, manteniéndolos en niveles óptimos, según se puede observar en la figura 4 , cuyo inicio fue de $146,20 \mathrm{mg} / \mathrm{dL}$ con disminución estadísticamente significativa $(\mathrm{p}=0,035)$, hasta 105,50 $\mathrm{mg} / \mathrm{dL}$ después de los tres meses de consumo. Resultados comparables a lo reportado por Alonso $^{9}$, quien encontró una disminución significativa $(\mathrm{p}=0,029)$ de TG séricos en un grupo de estudio con sobrepeso y obesidad después del consumo de un licuado de avena con manzana, semejante a lo reportado por Khoury ${ }^{11}$, encontrándose relación entre el consumo de avena sobre los niveles plasmáticos de lípidos, debido a la fibra soluble ( $\beta$-glucanos) e insoluble, que evita la absorción de los lípidos durante la digestión; asimismo, Thakur ${ }^{4}$ encontró relación entre el consumo de goma de linaza y la disminución de TG en sangre, por lo que esta variación estaría provocada por la fibra de avena y linaza de nuestra bebida. 


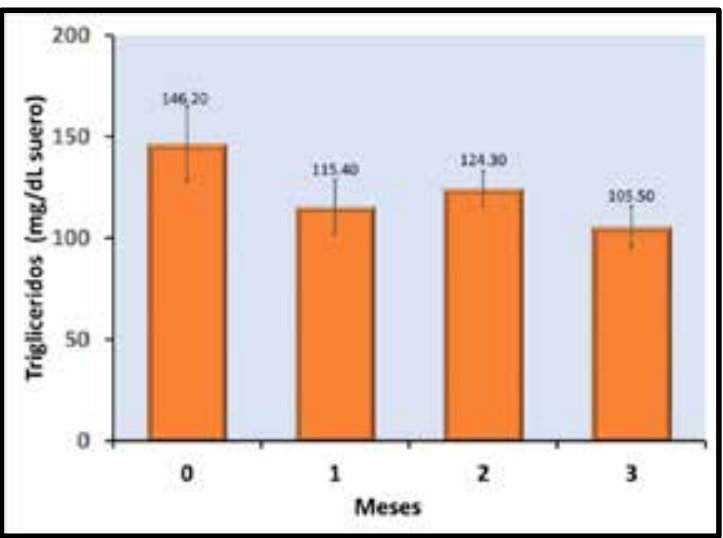

Figura 4. Evolución de los niveles séricos de triglicéridos del grupo de estudio con el consumo de la bebida durante tres meses.

Colesterol total: La comparación de muestras relacionadas en colesterol total cumplieron con los criterios de normalidad (S-W Inicio-1 mes =0,912, $\mathrm{p}=0,294$; S-W 1 mes-2 mes =0,970, $\mathrm{p}=0,890$; S-W 2 mes-3 mes $=0,877, \mathrm{p}=0,120$; S-W Inicio-Final $=0,966, \mathrm{p}=0,855$ ), con diferencia estadísticamente significativa entre el segundo y tercer mes, $t=2,852, p=0,019$, efecto de magnitud alta $(\mathrm{d}=0,902)^{9}$.

Pero, la disminución entre el inicio y final no muestra diferencia estadísticamente significativa $(\mathrm{p}=0,216) \mathrm{de} 139,40 \mathrm{mg} / \mathrm{dL}$ a $129,10 \mathrm{mg} / \mathrm{dL}$, respectivamente.

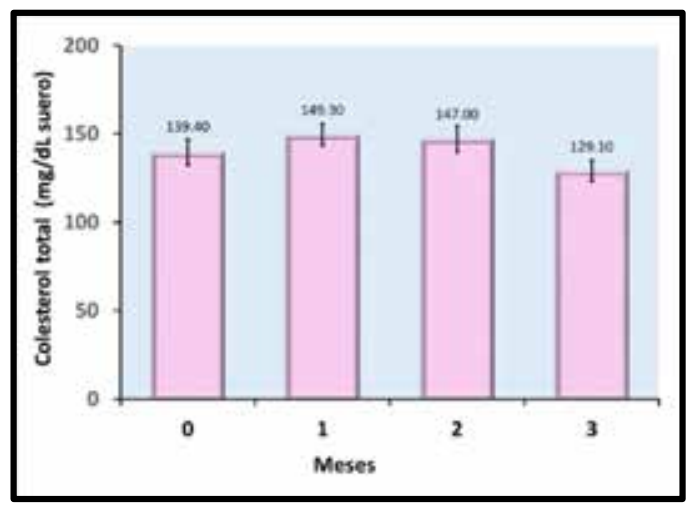

Figura 5. Evolución de los niveles séricos de colesterol total del grupo de estudio con el consumo de la bebida durante tres meses. 
Sin embargo, las concentraciones se mantuvieron en niveles óptimos ( $140 \mathrm{mg} / \mathrm{dL} \mathrm{a}<200 \mathrm{mg}$ / dL). Comparable al estudio de Alonso ${ }^{9}$, donde no se encontró una disminución significativa $(\mathrm{p}=0,173)$ de los niveles séricos de colesterol total y con lo reportado por Colonia ${ }^{12}$, en la evaluación de consumo de linaza sobre el perfil lipídico, tampoco encontró diferencia significativa para los niveles de colesterol total en un grupo de adultos aparentemente sanos, mientras que Thakur ${ }^{4}$ encontró una disminución significativa de colesterol total después de un consumo de goma de linaza. Podemos suponer que nuestros resultados sean debido a la variabilidad en la dieta del grupo de estudio; otra razón, puede ser, que a diferencia de los TG, que son usados como fuente de energía, el colesterol, los fosfolípidos y derivados de ellos, se utilizan en todo el organismo para llevar a cabo otras funciones, Guyton ${ }^{13}$.

Colesterol HDL: La comparación de muestras relacionadas en HDL cumplieron con los criterios de normalidad, ( $\mathrm{S}-\mathrm{W}$ Inicio-1 mes $=0,967, \mathrm{p}=0,858$; $\mathrm{S}-\mathrm{W} 1$ mes-2 mes $=0,865$, $\mathrm{p}$ $=0,087$; S-W 2 mes-3 mes $=0,942, \mathrm{p}=0,575 ; \mathrm{S}-\mathrm{W}$ Inicio-Final $=0,945, \mathrm{p}=0,605)$. Existe diferencia estadísticamente significativa solo entre el inicio del estudio y el primer mes, $\mathrm{t}=$ $2,659, p=0,026$, con efecto de magnitud alta $(d=0,841)^{10}$; pero, entre el inicio y el final se presentó un efecto de magnitud mediana $(\mathrm{d}=0,624) 10$; podemos decir que el consumo de la bebida contribuye medianamente en aumentar los niveles de HDL y equilibrar a los niveles óptimos en sangre (figura 6).

Sin embargo, al no ser estadísticamente significativo $(\mathrm{p}=0,080)$, nos indica en el consumo de la bebida no presenta un efecto significativo en los niveles séricos de HDL-col.

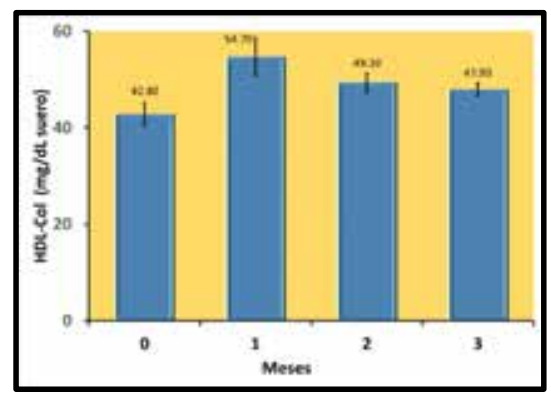

Figura 6. Evolución en los niveles séricos de colesterol HDL del grupo de estudio con el consumo de la bebida durante tres meses.

Colesterol LDL: La comparación de muestras relacionadas en LDL, cumpliendo los criterios de normalidad (S-W Inicio-1 mes =0,925, $\mathrm{p}=0,400$; S-W 1 mes-2 mes =0,945, p =0,613; $\mathrm{S}-\mathrm{W} 2$ mes-3 mes $=0,860, \mathrm{p}=0,077$; S-W Inicio-Final $=0,938, \mathrm{p}=0,534)$. Solo entre el segundo y tercer mes se presenta diferencia estadísticamente significativa, $t=2,819$, $p$ $=0,020$, con efecto de magnitud alta $(\mathrm{d}=0,891)^{9}$, el consumo de la bebida no provocó disminución de LDL, aunque estos se mantuvieron en el rango óptimo.

La figura 7 muestra los niveles séricos de LDL, antes $68,3 \mathrm{mg} / \mathrm{dL}$ y al final $60,1 \mathrm{mg} / \mathrm{dL}$ disminución no significativa estadísticamente $(\mathrm{p}=0,232)$, resultados comparables a los de Colonia $^{12}$. 


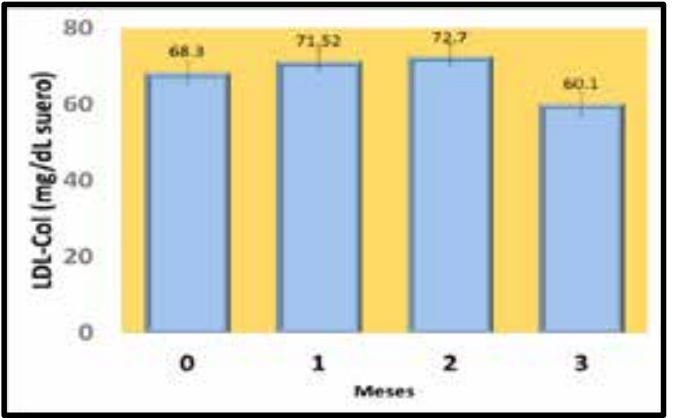

Figura 7. Evolución en los niveles séricos de colesterol LDL del grupo de estudio con el consumo de la bebida durante tres meses.

Glucosa: La comparación de los resultados aplicando t-student para muestras pareadas, esta comparación demostró cumplir con los criterios de normalidad ( $\mathrm{S}-\mathrm{W}$ Inicio-1 mes $=0,918$, $\mathrm{p}=0,338 ; \mathrm{S}-\mathrm{W} 1$ mes-2 mes $=0,885, \mathrm{p}=0,148 ; \mathrm{S}-\mathrm{W} 2$ mes-3 mes $=0,951, \mathrm{p}=0,680 ; \mathrm{S}-\mathrm{W}$ Inicio-Final $=0,939, \mathrm{p}=0,538$ ).

Existe diferencia estadísticamente significativa entre el inicio y el final del estudio, $t=4,342$, $\mathrm{p}=0,002$, con efecto de magnitud alta $(\mathrm{d}=1,373)^{10}$, y entre meses, excepto entre el primer y segundo mes, $t=0,288, \mathrm{p}=0,288$; podemos decir que el consumo de la bebida tiene efecto en la disminución de la glicemia y su relación en niveles óptimos.

En la figura 8 se observan los niveles séricos de glucosa desde antes del consumo de la bebida con $90,70 \mathrm{mg} / \mathrm{dL}$, con disminución estadísticamente significativa $(\mathrm{p}=0,002)$ al término del estudio a $71,90 \mathrm{mg} / \mathrm{dL}$. Resultados comparables a lo reportado por Biôrklund ${ }^{14}$ en glicemia (postpandrial) por consumo diario de $5 \mathrm{~g}$ de $\beta$-glucanos de avena en una bebida, por hombres y mujeres con colesterol medianamente elevado; y a Thakur ${ }^{4}$, quien encontró disminución significativa $(p=0,03)$ de glicemia en ayunas por consumo de goma de linaza por un periodo de tres meses; efecto atribuido a la fibra de avena y linaza contenida en la bebida, que hace la digestión y absorción de carbohidratos más lenta por el revestimiento de la mucosa intestinal $^{4,14}$. El empleo de stevia, cero calorías, debido a los glucósidos (esteviósidos y rebaudiosidos) que no producen energía ${ }^{15}$, ayuda a generar una menor respuesta glicémica. 


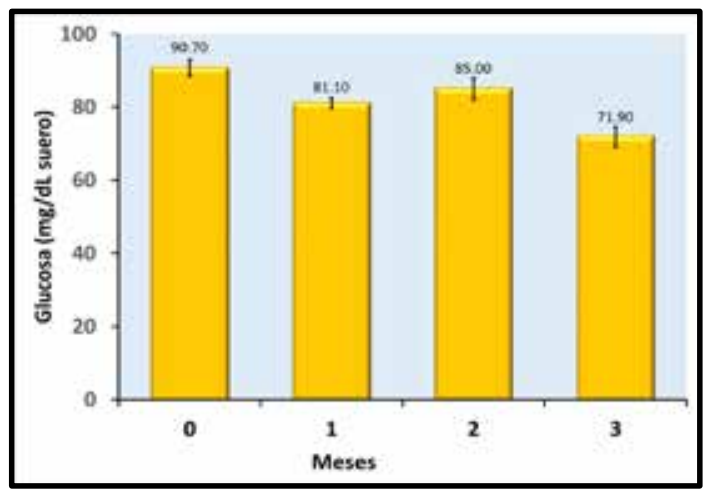

Figura 8. Evolución de la glicemia del grupo de estudio con el consumo de la bebida durante tres meses.

Hemoglobina: La comparación de los resultados aplicando t-student para muestras pareadas, demostrando que no cumplieron con los criterios de normalidad ( $\mathrm{S}-\mathrm{W}$ Inicio-1 mes $=0,717$, $\mathrm{p}=0,001 ; \mathrm{S}-\mathrm{W} 1$ mes-2 mes $=0,820, \mathrm{p}=0,025 ; \mathrm{S}-\mathrm{W} 2$ mes-3 mes $=0,658, \mathrm{p}=<.001 ; \mathrm{S}-\mathrm{W}$ Inicio-Final $=0,751, \mathrm{p}=0,004)$.

No se observaron diferencias estadísticamente significativas entre el inicio del estudio y el tercer mes, esto es debido a que los valores de hemoglobina se mantuvieron constantes, demostrando que la bebida no provocó cambios en la hemoglobina sérica (figura 9). Lo antes indicado es favorable para el estudio ya que tradicionalmente se ha considerado a la fibra dietética como inhibidor de la absorción de minerales ${ }^{16}$; en la absorción de hierro hemo y nohemo tiene lugar fundamentalmente en el duodeno ${ }^{17}$; únicamente el $10 \%$ del hierro ingerido se absorbe en este tramo del intestino ${ }^{18}$, la no disminución en los niveles de hemoglobina evidencia una absorción normal del hierro hemo, debido al medio ácido en el intestino que favorece la absorción de minerales en general, pues a $\mathrm{pH}$ bajo se mantienen en solución. El consumo de fibra soluble favorece a las bacterias beneficiosas en el tracto intestinal manteniendo un $\mathrm{pH}$ ácido en el medio, siendo ese el rol de la fibra de avena y linaza en la bebida. Asimismo, se ha documentado el papel estimulante que ejerce la vitamina $C$ en la absorción de hierro ${ }^{18,19}$, lo que se garantiza con la pulpa de frutas utilizada en nuestra bebida. 


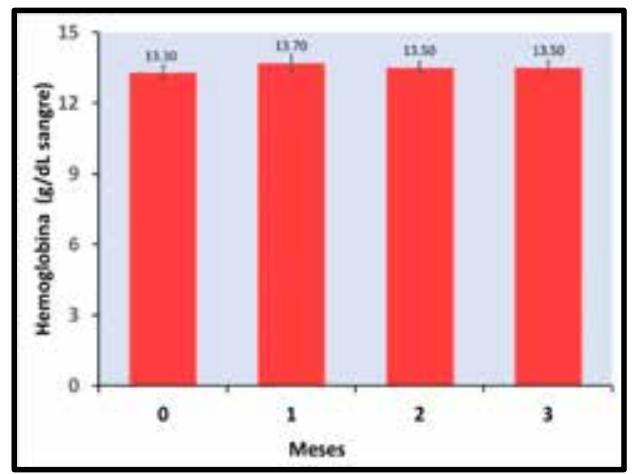

Figura 9. Evolución en los niveles séricos de hemoglobina del grupo de estudio con el consumo de la bebida durante tres meses.

Mediante este estudio podemos comprobar que hay resultados estadísticamente significativos en comparación del inicio y final del estudio en cuanto a los niveles séricos de triglicéridos y glucosa, la posible relación de este efecto puede ser atribuido al consumo de fibra de avena y linaza que ayuda a disminuir la absorción de lípidos y carbohidratos ${ }^{4}$. La fibra soluble, una vez que ingresa al estómago, enlentece el vaciamiento gástrico, origina distensión y aumenta la saciedad. En el intestino delgado también enlentece el tránsito. Esta fibra aumenta el espesor de la capa de agua que han de pasar los solutos para llegar al enterocito, de esta manera disminuye la absorción de glucosa, lípidos y aminoácidos ${ }^{20}$.

Los índices de colesterol total, HDL y LDL, fueron variables durante el estudio. Asimismo, como variables son las investigaciones al respecto, probablemente por falta de restricciones en la dieta del grupo de estudio o que los evaluados no fueron hiperlipidémicos, o sufrían alguna enfermedad, por ejemplo Thakur ${ }^{4}$, encontró una disminución de colesterol total y glucosa por consumo de goma de linaza, en pacientes con diabetes tipo 2. En nuestro caso, probablemente, debido a que encontramos al grupo de estudio entre los niveles óptimos de colesterol total, HDL y LDL antes del inicio del consumo de la bebida, estos se mantuvieron en el tiempo. Por otro lado, el exceso de carbohidratos en la dieta se convierte en triglicéridos, que se depositan en el tejido adiposo, lo cual está relacionado al aumento del IMC y perímetro de cintura; sin embargo, estos índices antropométricos, relacionados al sobrepeso y la obesidad, no mostraron disminución significativa, probablemente debido a que no solo es necesario con una dieta saludable sino también actividad física diaria ${ }^{8}$ y en el estudio realizado no se hizo una restricción a la dieta del grupo de estudio, ni hubo influencia de actividad física incorporada. Finalmente, se comprobó que la bebida posee propiedad hipolipemiante en cuanto a triglicéridos e hipoglicemiante, con resultados estadísticamente significativos, $\mathrm{p}=0,035 \mathrm{y} \mathrm{p}=0,002$, respectivamente. 


\section{CONCLUSIONES}

La bebida evaluada evidenció un efecto hipolipemiante en los niveles séricos de triglicéridos, siendo la variación estadísticamente significativa. El consumo de la bebida evaluada provocó un efecto hipoglicemiante, estadísticamente significativo. No se evidenciaron cambios estadísticamente significativos en las medidas antropométricas, ni en los de hemoglobina.

\section{AGRADECIMIENTOS}

- Al Vicerrectorado de Investigación de la Universidad Nacional Mayor de San Marcos por el financiamiento en la investigación

- Al laboratorio de Investigación de Bioquímica de la Facultad de Farmacia y Bioquímica de la Universidad Nacional Mayor de San Marcos por el uso de sus instalaciones.

- Al Servicio Académico Asistencial de Análisis Clínicos de la Facultad de Farmacia y Bioquímica de la Universidad Nacional Mayor de San Marcos.

\section{REFRERENCIAS BIBLIOGRÁFICAS}

1. Álvarez D, Sánchez J, Gómez G, Tarqui C. Sobrepeso y obesidad: Prevalencia y determinantes sociales del exceso de peso en la población peruana (2009-2010). Rev Perú Med Exp Salud Pública. 2012; 29(3): 303-13.

2. Troyo P. Obesidad y dislipidemias. Gac Méd Méx. 2004; 140(Supl. 2): S49-S58.

3. Matos A, Chambilla E. Importancia de la Fibra Dietética, sus Propiedades Funcionales en la Alimentación Humana y en la Industria Alimentaria. Rev Investg Cienc Tecnol Aliment. 2010;1(1): 4-17.

4. Thakur G, Mitra A, Pal K, Rousseau D. Effect of flaxseed gum on reduction of blood glucose \& cholesterol in Type 2 diabetic patients. Int J Food Sci Nutr. 2009; 60(Suppl 6): 126-36

5. INDECOPI. NTP 203.110:2009 .Jugos, néctares y bebidas de fruta. Requisitos.

6. FAO/WHO. Codex Alimentarius. Zumos, jugos de fruta y productos afines. Segunda Edición. Roma: FAO; 1992.

7. Horwitz W, Latimer G. Association of Official Analytical Chemists (AOAC). Official Methods of Analysis. Gaithersburg: Association of Officiating Analytical Chemists; 2007.

8. Organización mundial de la salud (OMS). Obesidad y sobrepeso. [Internet] Junio, 2016. [Ciltado 03 oct 2016]. Disponible en: http://www.who.int/mediacentre/factsheets/ fs311/es/

9. Alonso J, Bautista R, Calzada D, Gutiérrez M, Mendoza R. Efecto del licuado de avena con manzana sobre dislipidemias y sobrepeso-obesidad. CuidArte [Internet] 2012 [Citado 07 oct 2016]; 1(2): 24-33. Disponible en: http://journals.iztacala.unam.mx/ index.php/cuidarte/article/viewArticle/217 
10. Cohen J. Statistical power analysis for the behavioral sciences. 2a ed. Hillsdale, NJ: Lawrence Erlbaum. 1988.

11. El Khoury D, Cuda C, Luhovyy BL, Anderson GH. Beta Glucan: Health Benefits in Obesity and Metabolic Syndrome. J Nutr Metab. 2012; 2012:851362. Doi:10.1155/2012/851362.

12. Colonia A. Efecto del consumo de linaza (Linum usitatissimum) sobre el perfil lipídico de adultos aparentemente sanos. [Tesis] Lima: Universidad Nacional de San Marcos, Facultad de Medicina Humana; 2011.

13. Guyton A.C., Hall J.E. Tratado de Fisiología Médica. Madrid: Elsevier; 2006.

14. Biörklund M, Van Rees A, Mensink RP, Onning G. Changes in serum lipids and postprandial glucose and insulin concentrations after consumption of beverages with beta-glucans from oats or barley: a randomised dose-controlled trial. Eur J Clin Nutr. 2005; 59(11): 1272-1281.

15. Gupta E, Purwar S, Sundaram S, Rai G. Nutritional and therapeutic values of Stevia rebaudiana: A review. J Med Plants Res. 2013; 7(46): 3343-3353.

16. Toxqui L, de Piero A, Courtois V, Bastida S, Sánchez-Muniz FJ, Vaquero MªP. Deficiencia y sobrecarga de hierro; implicaciones en el estado oxidativo y la salud cardiovascular. Nutr Hosp. 2010; 25(3): 350-365.

17. Sharp P, Srai SK. Molecular mechanisms involved in intestinal iron absorption. World J Gastroenterol. 2007; 13(35): 4716-4724.

18. Slavin JL. Position of the American Dietetic Association: health implications of dietary fiber. J Am Diet Assoc. 2008; 108(10): 1716-31.

19. Debnath P, Dey P, Chanda A, Bhakta T. A Survey on Pineapple and its medicinal value. SAJP. 2012; 1(1):24-29.

20. Escudero E, Gonzales P. La fibra dietética. Nutr Hosp. 2006; 21(Supl.2): 61-72. 